\title{
Study of Protein Targeting and Mislocalization in Rod Photoreceptors
}

Asael Nunez ${ }^{1}$, Shimpei Takita ${ }^{1}$, Sanae Imanishi ${ }^{1}$, and Yoshikazu Imanishi ${ }^{1}$

${ }^{1}$ Department of Ophthalmology, Eugene and Marylin Glick Eye Institute, Indiana University

School of Medicine

The photoreceptor outer segment (OS) is a highly specialized organelle for light absorption. Precise localization of OS resident proteins is important for photoreceptor function. Molecular mechanisms underlying OS targeting of proteins and their mislocalization, which frequently causes inherited retinal degeneration, have been intensely investigated. Rhodopsin, a major protein of the rod OS, is often mislocalized to the inner segment (IS) plasma membrane of rod photoreceptors in retinal degeneration patients. In the Xenopus laevis model of retinitis pigmentosa, we previously found that $\mathrm{Na}^{+} / \mathrm{K}^{+}$-ATPase (NKA), a major IS protein, was downregulated. The Imanishi lab recently created a novel retinitis pigmentosa mouse model carrying the Q344ter rhodopsin gene mutation, which causes rhodopsin mislocalization to the rod IS plasma membrane. In this summer program, we examined whether this mouse model also displays reduced NKA expression in the rod IS's by immunohistochemistry at postnatal day 30. Although NKA was properly localized to the IS plasma membrane, expression of NKA was reduced in mutant photoreceptors compared to wildtype cells. In the rod OS, activation of rhodopsin eventually leads to the closure of the cyclic nucleotide gated (CNG) channel, which consists of $\alpha$ and $\beta$ subunits. This channel localizes to the OS plasma membrane, and the $\mathrm{N}$ terminal proline-rich region (R) of the $\beta$ subunit (CNG $\beta 1$ ) may be important for its interaction with peripherin (PRPH2), another OS resident protein. Currently, it is not well understood whether this interaction is necessary for the proper localization of CNG $\beta 1$ to the OS plasma membrane. Using Xenopus as a model, we studied the role of the $\mathrm{N}$-terminal proline-rich region in properly localizing $C N G \beta 1$ to the OS plasma membrane by generating transgenic $\operatorname{CNG} \beta 1(\Delta R)$ tadpoles that expressed $C N G \beta 1(\Delta R)$ in rods under the control of a rhodopsin promoter. We found that $C N G \beta 1(\Delta R)$ properly localized to the OS plasma membrane. 